\title{
Analysis of a case of periodic alternating nystagmus
}

\author{
PETER RUDGE AND JOHN LEECH \\ From the MRC Hearing and Balance Unit, National Hospital, Queen Square, London
}

SYNOPSIS A patient is described in whom there was periodic deviation of the eyes in darkness associated with alternating nystagmus. An exactly similar situation occurs in normal man subjected to a pendular stimulus. It is proposed that in this patient there was alternating activity of the vestibular nuclei on either side of the brain-stem as a result of a removal of cerebellar inhibition, and that this caused periodic shifts in the null region of Daroff and Dell'Osso.

Periodic alternating nystagmus is a rare but well-recognized phenomenon. This report concerns a patient who developed an unusual form of this condition in which it is thought that there was a reversible cerebellar lesion. The mechanism of alternating nystagmus is discussed.

\section{CASE HISTORY}

(N.H. A79822) An 18 year old unmarried bank clerk presented at hospital with a one week history of vertigo, unsteadiness, nausea and vomiting after a febrile illness. These symptoms, which were exacerbated by head movement, had progressively worsened

(Accepted 3 November 1975.) before admission. There was no significant past or family history.

NEUROLOGICAL EXAMINATION ON ADMISSION The patient was afebrile but looked unwell. Examination of the cranial nerves revealed full extraocular movements, with an inconstant horizontal nystagmus, which periodically reversed. There was no positional nystagmus. The remainder of the cranial nerves were normal. The limbs were normal apart from minimal ataxia of the right arm on finger/nose/finger testing. Gait and stance were extremely unsteady and the patient veered to the right.

INVESTIGATIONS A lumbar pneumoencephalogram was normal. The CSF contained $0.25 \mathrm{~g} \cdot \mathrm{l}^{-1}$ protein,

a
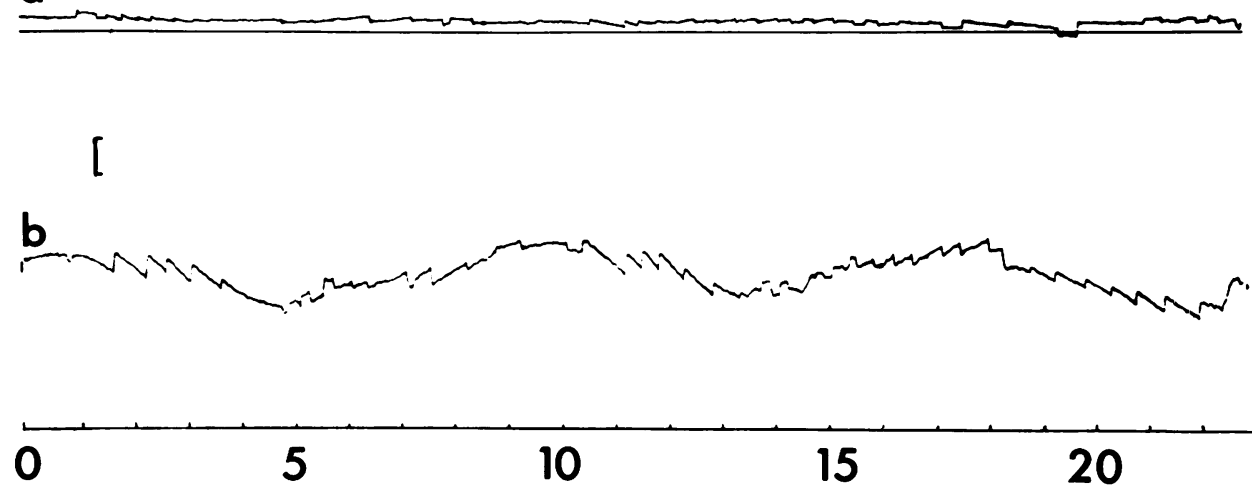

15

20

time (s)

FIG. 1 a. Nystagmographic trace of patient, eyes open, fixating target. Deviation to right upwards, to left downwards in this and all subsequent traces. b. Nystagmographic trace of patient with eyes deviated $30^{\circ}$ to left in darkness, eyes open. Vertical bar $10^{\circ}$ deviation. Time, seconds. 
with normal $\mathrm{IgG}$, and 6 lymphocytes $\mathrm{mm}^{-3}$. The EEG revealed paroxysmal bilateral slow wave activity of no fixed periodicity, and the visual evoked potential to a patterned stimulus was normal from each eye. Otological examination was normal apart from the caloric tests (Fitzgerald and Hallpike) which gave exaggerated responses to hot and cold irrigation without directional preponderance. The audiogram was normal.

ELECTRONYSTAGMOGRAPHIC STUDIES The nystagmus was recorded electronystagmographically with d.c. amplification using an Elema Schonander Mingograph 61 ink jet recorder. The resultant tracings provide a record of the nystagmus and any concomitant gaze deviation in the horizontal plane.

At the first examination a horizontal nystagmus was apparent on primary gaze with the eyes open. It was direction changing, beating alternately to left and to right with a periodicity of approximately 10 seconds, as shown in Fig. 1a. When the patient was asked to fixate upon a small illuminated target $30^{\circ}$ to the right, the character of the nystagmus changed to a fine nystagmus to the left interspersed with a few small saccades. Upon lateral deviation $30^{\circ}$ to the left, however, only small saccadic movements were recorded.

When the patient was asked to close her eyes and attempt to maintain the same gaze deviation that was present with the eyes open, a curious pendular undulating movement occurred. Superimposed upon this was a nystagmus that beat sometimes to the left and sometimes to the right. There was no definite periodicity of the nystagmus reversals and it was present in all attempted deviations of gaze.

The most remarkable feature of the nystagmus became apparent when recordings were carried out with the eyes open in total darkness while attempting to maintain gaze deviation. This provoked a rhythmic lateral deviation of the eyes with a total amplitude of some $20^{\circ}$ and a regular periodicity of approximately 10 seconds. Upon this was superimposed an alternating nystagmus, the slow phase of which was always in the direction of movement of the eyes. In contrast with other forms of spontaneous vestibular nystagmus, the present type was minimal at the peaks of the excursions, when the eyes were stationary, and greatest at the point of maximal eye velocity. This feature was particularly marked when attempting to maintain gaze deviation $30^{\circ}$ to the left (Fig. 1b). These findings were confirmed by direct observation of the patient's eyes with an infra-red viewer (Dix and Leech, 1974). This also demonstrated that there was no spontaneous head movement or inadvertent eye closure.

Ocular pursuit movements were studied using a

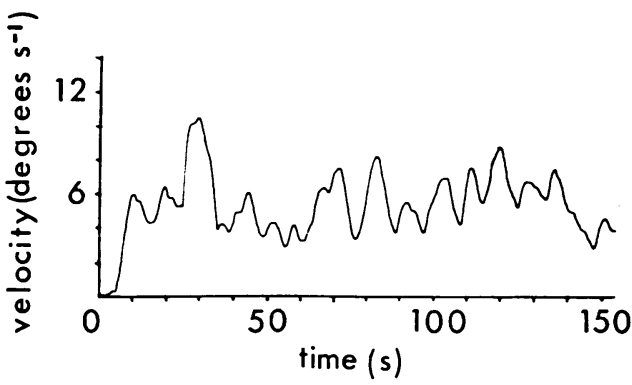

FIG. 2 Slow phase plot of optokinetic nystagmus. Vertical axis, velocity degrees per second. Horizontal axis, time seconds.

small illuminated target which could be moved through an arc of $60^{\circ}$ at any predetermined velocity from 10 to $40^{\circ} \mathrm{s}^{-1}$. In order to establish whether or not peripheral retinal feedback exerted any influence upon the ocular pursuit movements, testing was carried out with the target viewed both against a black and white striped background and in total darkness. In fact, there was no noticeable difference in the response pattern under the two test conditions,

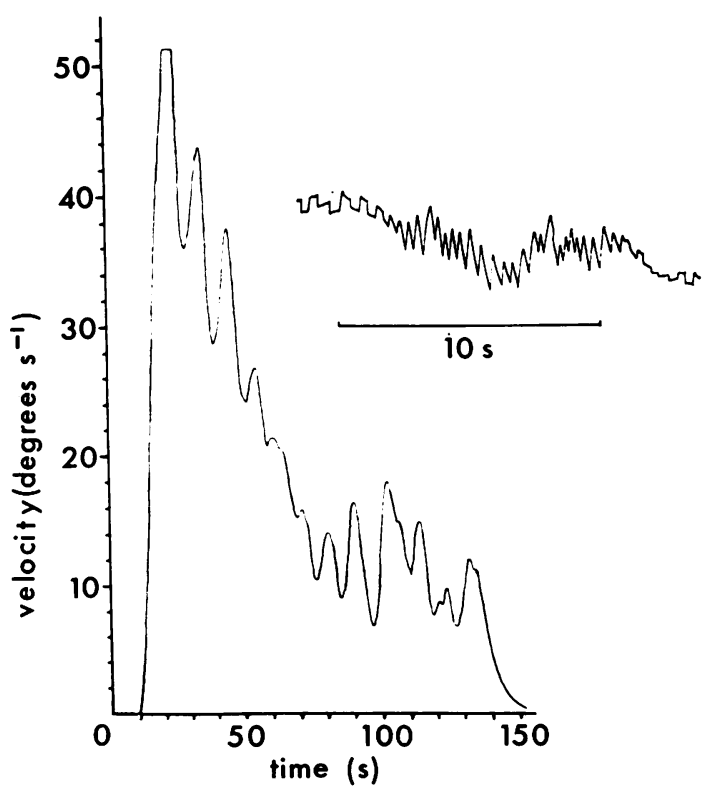

FIG. 3 Slow phase plot of rotation induced nystagmus. Vertical axis, velocity degrees per second. Horizontal axis, tim? seconds. Insert: example of single period of nystagmographic trace. 
following movements being full and interrupted only by small superimposed saccades. Recordings of the optokinetic responses were carried out using both a small optokinetic drum and a large servo-mechanically controlled drum that encircled the patient and filled the visual fields. The nystagmic response was fed into a slow phase velocity 'on line' computing system and displayed on an $\mathrm{X}-\mathrm{Y}$ plotter (Trinder, 1975). A typical response is shown in Fig. 2, where it will be seen that the velocity of the slow phase of the induced nystagmus fluctuates with a periodicity almost identical with that of the spontaneous nystagmus. This superimposition of the spontaneous movements of the eyes upon induced nystagmus responses was similarly present in the responses to rotational tests carried out 14 days later. In these, using an electrically controlled rotating chair (Byford et al., 1953), the patient was impulsively rotated to, and stopped from, a constant velocity of $40^{\circ} \mathrm{s}^{-1}$, the nystagmus being recorded with the eyes open in total darkness. The slow phase plot is shown in Fig. 3, together with examples of the nystagmic traces over a single period. Once again it will be seen that the same fluctuation occurred as in the case of the optokinetic responses, with a period comparable with that of the spontaneous nystagmus. Other electro-nystagmographic studies carried out on this occasion were substantially the same as those 14 days earlier.

Some two and a half months later, however, a further full electro-nystagmographic examination recorded only mild saccadic movements, mainly in darkness, with no evidence of the periodicity present on the previous occasions. At this time the patient was symptomatically well and no neurological signs were detected on clinical examination.

\section{DISCUSSION}

The aetiological diagnosis in this patient is unclear. It was considered that she suffered either from an encephalitic illness or from an episode of demyelination. The investigations were unhelpful in coming to a conclusion. However, the disorder of eye movement is of particular interest.

The abnormality in the patient might justifiably be called periodic alternating nystagmus in that there was a relatively regular periodicity to the nystagmus. However, this term is not clearly defined. Many types of nystagmus change

a
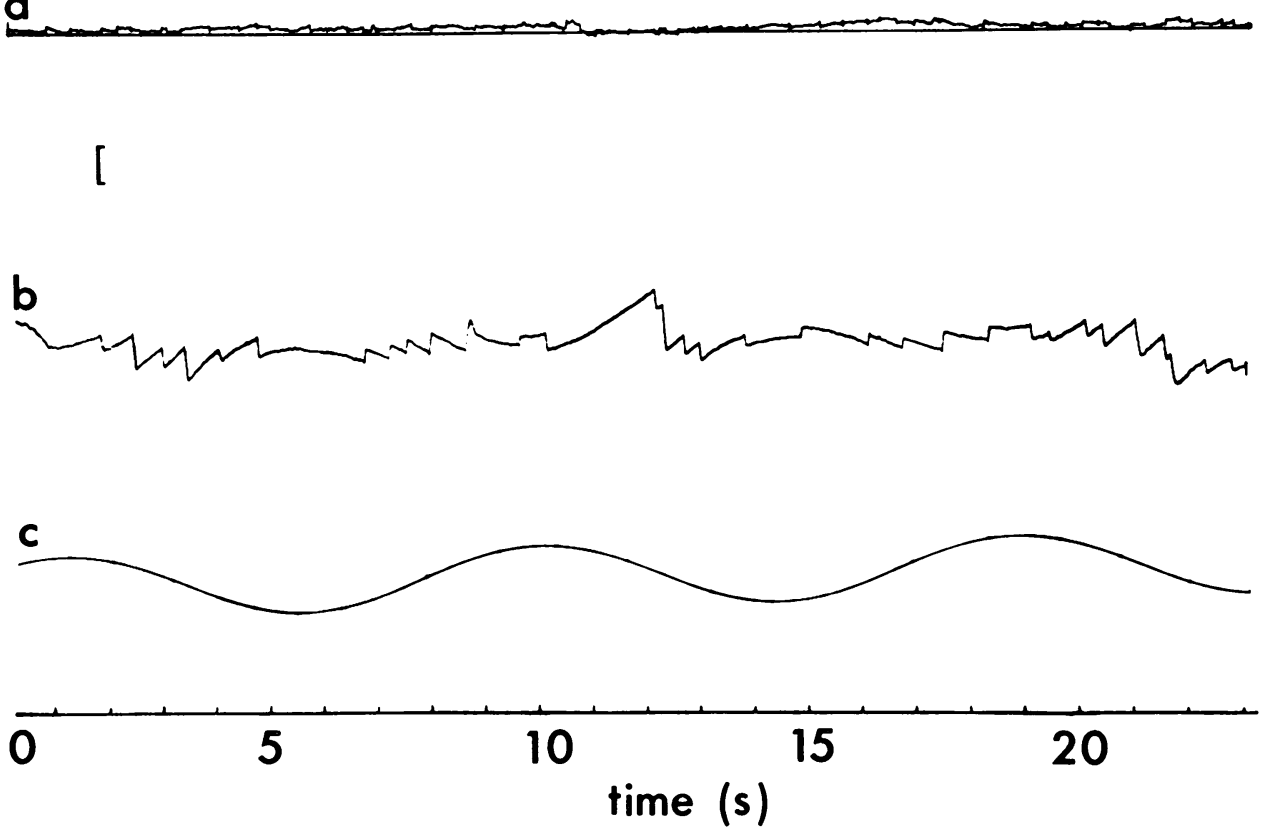

FIG. 4 a. Nystagmographic trace of normal person subjected to pendular stimulus, eyes open, fixating target. b. Nystagmographic trace of normal person subjected to pendular stimulus, eyes open, in dark with no fixation. c. Chair displacement. Vertical bar $10^{\circ}$ deviation. Time, seconds. 
direction-for example, congenital nystagmus, latent nystagmus, and rebound nystagmus, but it would not be of value to use the term periodic alternating nystagmus to describe all of these. Davis and Smith (1971) have defined periodic alternating nystagmus as a condition in which the periodicity of the nystagmus is essentially constant, of one to six minutes' duration, and with a 20 second quiescent interval. Not all authorities agree with such narrow limits (Collard, 1973). The present patient does not fulfil Davis and Smith's criteria and the abnormality of eye movement was more complex than simple alternating nystagmus. Thus the eyes deviated rhythmically by about $10^{\circ}$ to either side in the dark with a periodicity of approximately 10 seconds. Associated with this deviation was nystagmus which reversed with each oscillation and which was maximal at the point of minimal deviation. The oscillation and nystagmus were inhibited by fixation. What is the mechanism of these abnormalities?

We propose that a vestibular derangement is the cause of the abnormalities seen. In normal man subjected to a horizontal pendular stimulus in the dark, there is a periodic deviation of the eyes and alternating nystagmus (Fig. 4b). The nystagmus is identical with that seen in the present patient (compare Fig. 1b with Fig. 4b). Likewise in both, fixation abolished the deviation and markedly inhibited the nystagmus (Figs la and 4a). Thus it can be seen that alternating stimulation of the vestibular nuclei is a sufficient cause of the abnormalities observed in this patient. Furthermore, Jung and Kornhuber (1964) have postulated that a state of vestibular hyperresponsiveness is the basis of alternating nystagmus. By what mechanism does this activity alternate?

Perkel and Moloney (1974) pointed out that there are two basic mechanisms by which alternating activity can occur in the nervous system-namely, the oscillator hypothesis and the network hypothesis. In the former, individual cells have specialized membranes that rhythmically depolarize, while, in the latter, cells without this property are connected in such a way that they can excite each other periodically. It is the latter mechanism that is relevant to the present case. Perkel and Moloney have shown that if two neurones are connected by inhibitory synapses the phenomenon of postinhibitory rebound of excitability can cause alternating activity between the two cells. If neither cell is normally active - that is, they are not pacemaker cells-an additional input is necessary to start the activity which is then selfperpetuating. If the additional input be inhibitory, the initial stimulation is by the mechanism of post-inhibitory rebound. The pattern of alternation can be varied depending upon the intensity and the time of this input.

This model has particular relevance to an interesting suggestion put forward by Daroff and Dell'Osso (1974) that the basic abnormality in periodic alternating nystagmus is a periodic shift of the null region. This region is that where the drift forces upon the eyes are equal and the nystagmus is minimal. If during the course of optic fixation in the primary position of gaze this null region moves-for example, to the leftthen in this situation the eyes become effectively, though not actually, deviated to the right. The result will be a tonic deviation of the eyes to the left towards the null point with corrective movements taking the eyes back onto the target, bringing about a nystagmus with the fast phase to the right. With movement of the null point to the right, the nystagmus will reverse and beat to the left. In this way, although the gaze remains directed to a target in the primary position of gaze, the presumed periodic shift of the null point will give rise to a periodic alternating nystagmus which is maximal when the null point reaches its widest excursion and minimal when it passes through the midline.

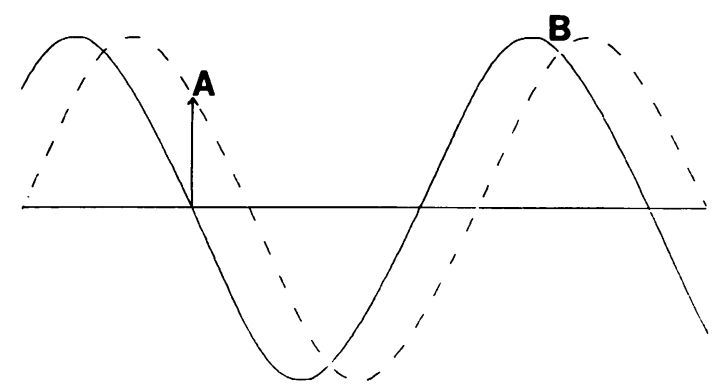

FIG. 5 Hypothetical deviation of null point (--) and eyes (-). A: point of maximum separation, B: point of coincidence of null and eye position. 
This applies to gaze restrained by optic fixation. With gaze unrestrained, as in our recordings in darkness, it is to be expected that the eyes will tend to take up the position of the null point executing periodic excursions which reflect its shifting position. In consequence, it can be argued that the eyes will maintain a condition of tonic neutrality and hence no nystagmus should result. It is, however, more than likely that a phase lag will exist between eye position and null point as illustrated in Fig. 5 in which the full line is taken to indicate the former, the interrupted line, the latter. In this event, the tonic forces giving rise to nystagmus and represented by the separation of the two curves will be maximal at $A$ where the eyes cross the midline and zero after the peak of the excursion of the eyes at B where the two curves cross. This, of course, is precisely the pattern of response which we have observed. Moreover, since similar considerations apply to the mechanism underlying pendular induced nystagmus, the demonstrable identity of the two is not surprising.

To this extent, therefore, our own studies are consistent with the null point hypothesis. It remains to be explained why the null point should move. It is proposed that the position of the null point is determined by the tonic activity of the vestibular nuclei. This activity is normally equal and opposite in direction from each side of the brain-stem. We suggest that Perkel and Moloney's model of alternating activity can be applied directly to the vestibular nuclei.

It is known that there are extensive commissural connections between the vestibular nuclei, particularly the superior and descending nuclei, and probably also the medial nuclei (Brodal, 1972). Some of these connections are inhibitory and monosynaptic (Shimazu, 1972). Such an interconnected system is similar to one of the alternating models proposed by Perkel and Moloney. Furthermore, it is known that there are inhibitory connections from the cerebellum to the vestibular nuclei especially from the flocculus, nodulus, and the spinal (vermis) part of the cerebellum (Walberg, 1972). An abnormality in such connections could initiate alternating activity of the vestibular nuclei and this rhythmical activity would be similar to that induced by pendular movement of the normal person.

There is some pathological evidence that a lesion of the cerebellum or its connections to the vestibular nuclei can cause periodic alternating nystagmus. Thus, Towle and Romanul (1970) described a patient with a cerebellar cyst in which periodic alternating nystagmus was apparent but in this case there was brain-stem compression. More recently, Keane (1974) has described a patient with multiple sclerosis who had periodic alternating nystagmus in whom there were essentially symmetrical lesions in the medial and inferior vestibular nuclei and in the inferior cerebellar peduncles. He proposed that the symmetry of the lesions was an important factor in the pathogenesis of periodic alternating nystagmus and agrees with Kornhuber that the removal of the inhibitory influences from the vestibular nuclei is the basic abnormality in this condition. In these cases, however, the eyes are not reported as deviating periodically as in the present patient. What evidence is there of such damage in the cerebellum or its connections in the present case?

The patient had a markedly ataxic gait with little evidence of ataxia in the limbs, a clinical picture compatible with a lesion of the vermis. The caloric responses were symmetrically brisk and of large amplitude, which is consistent with the removal of cerebellar inhibition from the vestibular nuclei. Similarly, the response to rotation was pronounced. Finally, small saccadic movements were prominent and nystagmus opposite in direction to that of gaze - that is, against Alexander's law-was found, both these abnormalities having been frequent findings in this laboratory in cerebellar disease.

There is, thus, some evidence of a lesion of the cerebellar system and this could have resulted in removal of inhibitory influences from the vestibular nuclei and therefore cause alternating activity of these nuclei. Such activity would have important implications.

For example, periodic activity originating in the vestibular nuclei would presumably have a marked effect upon induced eye movements. A visual stimulus causing optokinetic nystagmus would have this periodic activity superimposed. This can be seen in the plot of the slow phase of the optokinetic nystagmus in which there are periodic fluctuations. This periodicity is of the same frequency as that of the spontane- 
ous nystagmus. Similarly, nystagmus induced through semicircular canal stimulation by rotation of the patient fluctuated periodically. The slow phase plot again demonstrated the same periodicity as the spontaneous nystagmus. Both of these observations are consistent with the proposed abnormality of vestibular function. The likeness to nystagmus induced in a normal subject by the pendular motion suggests that this is indeed the physiological basis of the alternating nystagmus in the present patient.

In conclusion, we propose that, in the patient described, there was a reciprocal activation between the vestibular nuclei on each side of the brain-stem as the result of a partial removal of the inhibitory influences due to a lesion in the cerebello-vestibular pathways. This activity caused a periodic shift of the null region, as proposed by Daroff and Dell'Osso, and resulted in periodic deviation of the eyes and the associated nystagmus. Optic fixation could to some extent override the abnormality but did not totally abolish the disorder of eye movement.

The authors wish to thank Dr J. D. Hood for his advice and criticism in the preparation of this paper.

\section{REFERENCES}

Brodal, A. (1972). Organisation of the commisural connections: anatomy. Progress in Brain Research, 37, 167-176.
Byford, G. M., Hallpike, C. S., and Hood, J. D. (1953). A new type of rotating chair for the investigation of semicircular canal function. Journal of Physiology, 123, 22P.

Collard, M. (1973). Les Syndromes Vestibulaires Centraux, pp. 75-76. Masson: Paris.

Daroff, R. B., and Dell'Osso, L. F. (1974). Periodic alternating nystagmus and the shifting null. Canadian Journal of Otolaryngology, 3, 367-371.

Davis, D. G., and Smith, J. L. (1971). Periodic alternating nystagmus. American Journal of Ophthalmology, 72, 757762.

Dix, M. R., and Leech, J. (1974). The use of infra-red light in the evaluation of vestibular disorders. Journal of Laryngology and Otology, 88, 199-206.

Jung, R., and Kornhuber, H. H. (1964). Results of electronystagmography in man: the value of optokinetic, vestibular, and spontaneous nystagmus for neurologic diagnosis and research. In The Oculomotor System, p. 445. Edited by M. B. Bender. Harper Row: London.

Keane, J. R. (1974). Periodic alternating nystagmus with downward beating nystagmus. Archives of Neurology, 30, 399-402.

Perkel, D. H., and Moloney, B. (1974). Motor pattern production in reciprocally inhibitory neurons exhibiting postinhibitory rebound. Science, 185, 181-183.

Shimazu, H. (1972). Organisation of commissural connections: physiology. Progress in Brain Research, 37, 177-190.

Towle, P. A., and Romanul, F. (1970). Periodic alternating nystagmus: first pathologically studied case. Neurology (Minneap.), 20, 408.

Trinder, E. (1975). Instrument for the on-line measurement of the slow phase of nystagmus. Aviation, Space and Environmental Medicine, 46, 441-444.

Walberg, F. (1972). Cerebello-vestibular relations: anatomy. Progress in Brain Research, 37, 361-376. 